\title{
Modality-specific dysfunctional neural processing of social-abstract and non-social-concrete information in schizophrenia
}

\author{
Yifei He ${ }^{\mathrm{a}, \mathrm{d}, *}$, Miriam Steines ${ }^{\mathrm{a}, \mathrm{d}}$, Gebhard Sammer ${ }^{\mathrm{b}}$, Arne Nagels ${ }^{\mathrm{c}}$, Tilo Kircher ${ }^{\mathrm{a}, \mathrm{d}}$, \\ Benjamin Straube ${ }^{\mathrm{a}, \mathrm{d}}$ \\ a Department of Psychiatry and Psychotherapy, Philipps-University Marburg, Marburg, Germany \\ ${ }^{\mathrm{b}}$ Cognitive Neuroscience at Centre for Psychiatry, Justus-Liebig University Giessen, Giessen, Germany \\ ${ }^{\mathrm{c}}$ Department of General Linguistics, Johannes-Gutenberg University Mainz, Mainz, Germany \\ ${ }^{\mathrm{d}}$ Center for Mind, Brain and Behavior-CMBB, Hans-Meerwein-Straße 6, 35032 Marburg, Germany
}

\section{A R T I C L E I N F O}

\section{Keywords:}

Social

Multimodal processing

mPFC

Gesture

Speech

Schizophrenia

\begin{abstract}
A B S T R A C T
Schizophrenia is characterized by marked communication dysfunctions encompassing potential impairments in the processing of social-abstract and non-social-concrete information, especially in everyday situations where multiple modalities are present in the form of speech and gesture. To date, the neurobiological basis of these deficits remains elusive. In a functional magnetic resonance imaging (fMRI) study, 17 patients with schizophrenia or schizoaffective disorder, and 18 matched controls watched videos of an actor speaking, gesturing (unimodal), and both speaking and gesturing (bimodal) about social or non-social events in a naturalistic way. Participants were asked to judge whether each video contains person-related (social) or object-related (nonsocial) information. When processing social-abstract content, patients showed reduced activation in the medial prefrontal cortex (mPFC) only in the gesture but not in the speech condition. For non-social-concrete content, remarkably, reduced neural activation for patients in the left postcentral gyrus and the right insula was observed only in the speech condition. Moreover, in the bimodal conditions, patients displayed improved task performance and comparable activation to controls in both social and non-social content. To conclude, patients with schizophrenia displayed modality-specific aberrant neural processing of social and non-social information, which is not present for the bimodal conditions. This finding provides novel insights into dysfunctional multimodal communication in schizophrenia, and may have potential therapeutic implications.
\end{abstract}

\section{Introduction}

In everyday social communication, humans encounter a diverse spectrum of inputs from multiple modalities (Holler and Levinson, 2019), which might be potentially difficult to process for patients with schizophrenia. These include non-linguistic stimuli from others' facial expressions (Gur et al., 2002; Vuilleumier et al., 2001), body movements, e.g., postures and gestures (He et al., 2020; Nagels et al., 2015b; Straube et al., 2013b), and linguistic stimuli in the form of auditory speech and written texts (Brown and Kuperberg, 2015). Importantly, the multimodal inputs comprise both social-abstract and non-socialconcrete information, and patients' social functioning heavily depends on the processing of these stimuli, which serves as the basis to further mentalize social intentions and to perform appropriate social interaction (Amodio and Frith, 2006). To date, however, it remains elusive if the processing of these social/non-social information in schizophrenia is impaired in schizophrenia in a multimodal context.

Social cognition is known to be impaired in schizophrenia (Green et al., 2015). Regarding the perception of social stimuli, in particular, prior research on patients' social perception dysfunction has focused on emotional perception of faces and voices (Anticevic et al., 2012; Witteman et al., 2012), whereas limited studies have investigated the perception of social-abstract information delivered via linguistic and gesture stimuli. In healthy participants, a seminal fMRI (functional magnetic resonance imaging) study has identified distinct brain regions for processing socially and non-socially relevant linguistic information (Mitchell et al., 2002): When participants were asked to judge whether visual word pairs are person- or object-related, person-related social stimuli activated the medial prefrontal cortex (mPFC), a crucial region forming the mentalizing network (Amodio and Frith, 2006; Frith and

\footnotetext{
* Corresponding author at: Department of Psychiatry and Psychotherapy, Philipps University Marburg, Rudolf-Bultmann-Str. 8, 35039 Marburg, Germany.

E-mail address: yifei.he@staff.uni-marburg.de (Y. He).
} 
Frith, 2006; Saxe and Powell, 2006; Van Overwalle, 2009). On the other hand, when processing linguistic stimuli about non-social-concrete content (e.g., words and sentences about objects), healthy participants activate regions including a left-lateralized network including the left the bilateral insula and the left parietal lobe (Binder et al., 2005; Chao et al., 1999).

Notably, in everyday life, both types of information are also commonly conveyed via non-linguistic channels such as manual gestures. For instance, even without speech, individuals can use a "be silent" emblematic gesture to deliver social information, which is commonly intransitive and symbolic; on the other hand, for describing concrete actions, transitive and object-related hand actions, i.e., pantomimes such as "hammering" are commonly used. Moreover, it is common to use both gesture and speech together: To ask someone else to stop, one can use a "stop" gesture (e.g., a front-facing, raised hand) together with its verbal counterpart. Emerging basic research has investigated if the processing of social-abstract and non-social-concrete information is modality-independent: for healthy populations, socialabstract information delivered by both auditory speech and manual gesture commonly activates the MPFC and the (IFG) and middle temporal gyri (Straube et al., 2010, 2013c)—regions that are typically activated for social cognition and abstract semantics (Binder et al., 2009; Van Overwalle, 2009). For processing non-social action and object information, literature also suggests that humans recruit modalityindependent regions, such as the lateral occipitotemporal cortex (LOTC) and the pre/postcentral gyri (Barsalou, 2008; Pulvermüller et al., 2005; Wurm and Caramazza, 2019).

The multimodal characteristics of social/non-social information processing, as well as its supramodal neural basis, may have profound implications in schizophrenia research. Schizophrenia is characterized by well-known deficits in the comprehension and production of speech (Bleuler, 1950; Kircher et al., 2002; Kuperberg et al., 2008; Meyer et al., 2020), as well as impairments in the perception and production of gesture (Matthews et al., 2013; Millman et al., 2014; Mittal et al., 2006; Nagels et al., 2019; Straube et al., 2013a, 2013b; Thakkar et al., 2014; Walther et al., 2015, 2013). Clinically, these two aspects may directly contribute to major symptoms of schizophrenia such as formal thought disorder and apraxia/catatonia respectively. They are also indicative of a range of other negative and positive symptoms of schizophrenia (Park et al., 2008; Walther et al., 2019), and are functionally highly relevant (Friedman et al., 2012; Walther et al., 2016). Yet, regarding the processing of social/non-social information, it remains unknown if both processes are impaired in schizophrenia irrespective of modality, or if the impairments are modality-specific. For the processing of socialabstract information, both linguistic (visual and auditory) and gesture (social emblems) have not been investigated in schizophrenia so far. However, studies employing non-semantic hand actions have shown that patients might be impaired for gesture perception in general (Thakkar et al., 2014; Walther et al., 2015). For the processing of concrete and non-social information, a previous study shows that patients are impaired in visually presented sentences (Kuperberg et al., 2008). Moreover, given potential dysfunctional processing of social or nonsocial information in schizophrenia, it remains unclear if these deficits could be potentially compensated by multimodal inputs containing both speech and gesture: Emerging literature suggests mutual facilitation between speech and gesture, at least for healthy populations (Cuevas et al., 2019; Drijvers et al., 2018; He et al., 2018b; Krahmer and Swerts, 2007; Wang and Chu, 2013).

To address these remaining research questions, we conducted the current study, presenting to patients with schizophrenia and matched controls with videos of an actor communicating in a spontaneous and naturalistic manner. In these videos, the actor performs either socialabstract (person-related) or non-social (object-related) content in different modalities, where social and non-social information is perceivable in gesture- and speech-only modalities. Similar to approaches from previous research (Mitchell et al., 2002; Straube et al., 2013c), we directly compared social vs. non-social videos to identify neural perception of social and non-social information in both auditoryspeech and visual-gesture modalities. Additionally, we showed to participants videos with bimodal inputs (actor both speaking and gesturing). Based on previous research, we hypothesized activation of the mPFC and a left frontal-temporal network (e.g., inferior frontal gyrus, middle temporal gyrus) for the processing of social-abstract information (Mitchell et al., 2002). For non-social-concrete information processing, we hypothesized left-lateralized regions including the lateral occipitotemporal cortex (LOTC), the superior temporal gyrus/sulcus (STG/STS), as well as pre/postcentral gyri forming the putative mirror neuron system (Järveläinen et al., 2004; Johnson-Frey, 2004; JohnsonFrey et al., 2003; Lingnau and Downing, 2015). We focused directly on group differences between a group of patients suffering from schizophrenia or schizoaffective disorder, and their age- and educationmatched controls: for social content, as patients are well-known for their social cognition impairments, we expected patients to show reduced activation in the $\mathrm{mPFC}$, irrespective of encoding modality (Frith, 2004); For non-social content, despite mixed findings from previous neuroimaging research on hand action observation on schizophrenia (Horan et al., 2014; Thakkar et al., 2014), following previous report on dysfunctional processing of non-social linguistic stimuli in schizophrenia (Kuperberg et al., 2008), we hypothesized neural modulation of the object-related regions for patients with schizophrenia for both gesture and speech modalities. Additionally, based on prior basic research on mutual facilitation between gesture and speech (Cuevas et al., 2019; see Holler and Levinson, 2019 for review), we hypothesized that the bimodal input could compensate for potential unimodal processing deficits, leading to improved performance in the patient group.

\section{Methods}

\subsection{Participants}

We summarized participants' demographic and clinical characteristics in Table 1. Healthy controls were recruited matching age, gender, and education to patients. Seventeen patients were recruited at the

Table 1

Demographic, medication, symptom, and neuropsychological measures.

\begin{tabular}{llll}
\hline & $\begin{array}{l}\text { Patients }(\mathrm{n}= \\
17)\end{array}$ & $\begin{array}{l}\text { Controls }(\mathrm{n}= \\
18)\end{array}$ & $\begin{array}{l}\text { Difference } \\
(p)\end{array}$ \\
\hline Age (years) & $33.12(12.35)$ & $\begin{array}{l}31.94(10.21) \\
13 / 5\end{array}$ & 0.76 \\
Gender male:female & $13 / 4$ & $12.72(1.36)$ & 0.1 \\
Education (years) & $11.82(1.77)$ & $26.17(9.89)$ & 0.14 \\
TMT A (seconds) & $31.49(10.73)$ & $52.93(19.58)$ & 0.13 \\
TMT B (seconds) & $68.56(37.8)$ & $8.05(2.43)$ & 0.87 \\
Digit Span forward & $7.94(1.75)$ & $6.61(2.50)$ & 0.73 \\
Digit Span backward & $6.35(1.93)$ & $28.5(3.79)$ & 0.8 \\
Verbal IQ & $28.8(5.25)$ & $1.14(0.19)$ & 0.048 \\
*Concretism & $1.38(0.45)$ & & \\
SAPS (global) & $15(6.89)$ & & \\
SAPS (composite) total & $22.35(18.08)$ & & \\
SAPS 1 (hallucinations) & $4.64(6.33)$ & & \\
SAPS 2 (Delusions) & $10.47(9.37)$ & & \\
SAPS 3 (Bizarre behavior) & $1.88(2.93)$ & & \\
SAPS 4 (Thought & $5.41(7.00)$ & & \\
$\quad$ disorder) & & & \\
SANS (global) & $9(6.02)$ & & \\
SANS (composite) total & $21.00(13.63)$ & & \\
SANS 1 (Flat affect) & $7.18(6.20)$ & & \\
SANS 2 (Alogia) & $3.29(4.37)$ & & \\
SANS 3 (Apathy) & $4.76(4.51)$ & & \\
SANS 4 (Anhedonia) & $4.35(4.44)$ & & \\
SANS 5 (Attention) & $1.59(2.58)$ & & \\
CPZ Equivalent & $562.52(372.63)$ & & \\
\hline
\end{tabular}

Values are presented as mean (SD). TMT: trail making test; CPZ: chlorpromazine. Asterisk * indicates significant difference between controls and patients $(\mathrm{p}<$ 0.05 , two-tail $t$-test). 
Department of Psychiatry and Psychotherapy at the Philipps University of Marburg, and were diagnosed according to ICD-10 with schizophrenia (F20.0, $\mathrm{n}=13$, and F20.3, $\mathrm{n}=1$ ) or schizoaffective disorder (F25.0, $\mathrm{n}=$ 2 , and F25.2, $\mathrm{n}=1$ ). Participants in both groups are native speakers of German, and have no knowledge of Russian language. All except one of the patients received antipsychotic treatment; six were additionally treated with antidepressive medication (For detailed medication at the time scanning, see Table S2). Positive and negative symptoms were assessed with the Scale for the Assessment of Positive Symptoms (SAPS) (Andreasen, 1984), and the Scale for the Assessment of Negative Symptoms (SANS) (Andreasen, 1981). Eighteen age-, gender-, and education-matched healthy participants with no history of any mental disorders were recruited from the same area. Exclusion criteria for both groups were brain injury and neurological or other medical diseases affected by brain physiology. In both groups, we conducted neuropsychological tests to assess working memory function, digit span, trail making (TMT), verbal IQ (MWT-B) (Lehrl, 1999), and metaphoric language processing (concretism, evaluated with the Proverb Interpretation Task) (Barth and Küfferle, 2001). These measures are reported in Table 1. We report, additionally, scores from word fluency test, as well as gesture production and perception (BAG, Brief Assessment of Gesture (Nagels et al., 2015a)) in the supplement (Table S1). All participants had normal or corrected-to-normal vision and hearing. Except for one control and one patient, all other participants are right-handed (Oldfield, 1971). All participants gave written informed consent prior to participation in the experiment and were compensated monetarily. The study was approved by the ethics committee of the School of Medicine, Philipps University Marburg.

\subsection{Materials and procedure}

We employed a content judgement paradigm from previous studies from our research group to investigate modality-specific processing of social/non-social information (He et al., 2015; Straube et al., 2013c, 2018), which has elicited dissociable but supramodal neural processing of social-abstract and non-social-concrete information in a healthy student sample (Straube et al., 2013c). Of note, the same fMRI dataset has been published with an unrelated research question (Wroblewski et al., 2020). We showed to participants five-second videos of an actor spontaneously communicating both social-abstract (S) and non-socialconcrete $(\mathrm{N})$ events in the following modalities: 1) incomprehensible Russian sentences with gestures. This is considered as a gesture-only (G) condition because social feature is only available to participants in the gesture form. 2) comprehensible German sentences (S) without any gestures. Additionally, we also showed to participants 3) German sentences with accompanying gestures as a bimodal input condition (B). A filler condition is also included with videos of incomprehensible Russian sentences with meaningless gestures. An example of both a social (S) and non-social $(\mathrm{N})$ bimodal videos is illustrated in Fig. 1A. For a complete list of all videos, please refer to Appendix in He et al. (2015).

\subsection{Procedure}

Altogether, 312 experimental video slips (26 videos per condition $\times$ 6 conditions $\times 2$ sets) were included in the study. For each participant, an experimental session comprised 182 videos from one set of videos (156 critical videos and 26 filler videos), and consisted of two 14-minute runs. Each run contained 91 trials with a matched number of items from each condition. The stimuli were presented in an event-related design in pseudo-randomized order. Within each trial, each video-clip was followed by a gray background with a variable duration of $2154-5846 \mathrm{~ms}$ (jitter average: $4000 \mathrm{~ms}$ ), as illustrated in Fig. 1B. Participants performed a content judgement task for each video (Mitchell et al., 2002; Straube et al., 2013c), indicating via button press (with their left hand) whether a stimulus was either person- or object-related. Participants were instructed to respond to the task as soon as they had decided on an
A

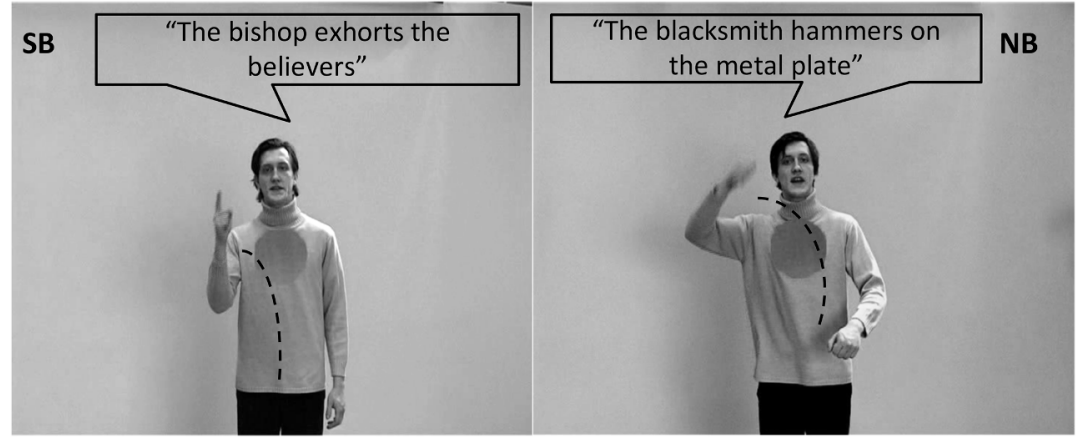

Fig. 1. Panel A: Picture illustration for socialabstract (S) and non-social-concrete $(\mathrm{N})$ videos in the bimodal condition (B). The same stimuli were also presented in two additional modalities: gestures with foreign Russian sentences (G) and German sentences without any gestures (S). For illustrative purposes, the spoken German sentences were translated into English, and all spoken sentences were written into speech bubbles. Panel B: Illustration of a sample trial. Participants performed a content judgment task for each video, indicating via button press whether a stimulus was either person- or objectrelated.

B

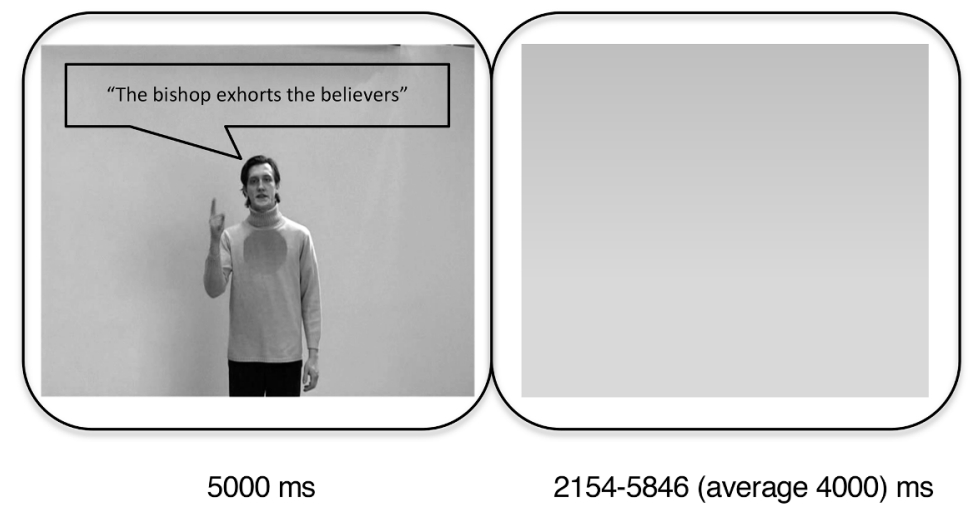


answer.

\section{4. fMRI acquisition and preprocessing}

All images were acquired using a 3 T MRI scanner (Siemens MRT Trio series). The functional images were obtained using a T2*-weighted echoplanar image sequence $\left(\mathrm{TR}=2 \mathrm{~s}, \mathrm{TE}=30 \mathrm{~ms}\right.$, flip angle $=90^{\circ}$, slice thickness $=4 \mathrm{~mm}$, interslice gap $=0.36 \mathrm{~mm}$, field of view $=230 \mathrm{~mm}$, matrix $=64 \times 64$, voxel size $=3.6 \times 3.6 \times 4.0 \mathrm{~mm}, 30$ axial slices orientated parallel to the AC-PC line, ascending order). Two runs of 425 volumes each were acquired during the experiment. Additionally, simultaneous EEG data from the participants were also collected for other analyses not relevant for the current study, and are therefore not further discussed here. MR images were preprocessed using the SPM12 software package (Statistical Parametric Mapping, Welcome Trust Center for Neuroimaging, London, UK) based on Matlab R2017a (version 9.2.0; MathWorks): after discarding the first five volumes to minimize T1-saturation effects, all images were spatially and temporally realigned, and normalized into the MNI space using the MNI template (resulting voxel size $2 \times 2 \times 2 \mathrm{~mm}$ ), smoothed ( $8 \mathrm{~mm}$ isotropic Gaussian filter), and high-pass filtered (cut-off period $128 \mathrm{~s}$ ).

\section{5. fMRI data analysis}

We performed statistical whole-brain analysis in a two-level, mixedeffects procedure. On the first level, single-participant BOLD responses were modeled by a design matrix comprising the onset time points of each event (critical word of each sentence as used in the previous eventrelated fMRI and EEG studies, e.g., (He et al., 2015, 2018a, 2018c; Straube et al., 2013c, 2018)), with a duration of $5 \mathrm{~s}$ for all experimental conditions. The micro-time onset was set to the average time bin ( 8 of 16) to align the onset vector to the slice in the middle of the brain. For all conditions, the duration of speech and gesture was used as parameters of no interests on a single trial level. Six movement regressors (three rotations and three translations) were entered in the single participant's model to account for movement-induced effects on fMRI results. HRF was defined as the canonical HRF. Contrasts images against implicit baseline for all experimental conditions were used as summary measures and were included in the between-group analysis. We applied a flexible factorial analysis of variance using condition as main effect. We applied a Monte-Carlo simulation to determine the cluster extent threshold to correct for multiple comparisons, (Slotnick, 2017; Slotnick et al., 2003), which has been used in comparable studies in our laboratory on social perception of multimodal stimuli (Nagels et al., 2015b). For all statistical comparisons, the whole-brain activation was simulated assuming a voxel type-I error activation of $\mathrm{p}<.05$, this revealed a cluster extent of 2268 contiguous resampled voxels as sufficient to correct for multiple comparisons at $\mathrm{p}<.0167$ (Bonferroni-corrected for three independent tests for interaction within three independent modalities, with $0.05 / 3$ ). We also reported in activation tables uncorrected cluster p-values and marked significance for cluster-level FWE correction. The reported voxel coordinates of activation peaks are located in MNI space. For the anatomical localization, functional data were referenced to the AAL toolbox (Tzourio-Mazoyer et al., 2002).

Firstly, we tested three-way interaction of group $\times$ modality $\times$ content. We then conducted, within each modality, interaction analyses to investigate group differences in the processing of social or non-social conditions, and masked the respective results based the contrast image from the first analysis, to reveal modality-specific group interaction. In addition, within each modality, for each group, we conducted pair-wise comparison between social and non-social conditions ( $>\mathrm{N}$ and $\mathrm{N}>\mathrm{S}$ ), for illustrating modality- and group-specific brain activations for either social or non-social information processing. In the end, following our hypotheses on bimodal enhancement for patients, within patients, we tested the interaction between modalities on social vs. non-social content processing, so as to reveal how bimodal stimuli might compensate potential neural processing deficits for patients with schizophrenia. Results of this analysis is reported in Supplement S3.

Based on the literature showing a potential relationship between symptom severity (especially negative symptoms) and social/non-social cognition (Mehta et al., 2014; Sergi et al., 2007), as well as gesture processing (Walther et al., 2019, 2013), for patients with schizophrenia, we conducted exploratory correlation analysis, probing for the potential relationship between clinical measures and brain activation in areas that are relevant to social/non-social information processing. To this end, spearman correlation analyses (uncorrected) were conducted between 1) parameter estimates from clusters showing significant group difference for either social or non-social conditions, 2) behavioral measures (reaction times and accuracy) for each experimental condition, and 3) scores from sum/general and subscales of SAPS and SANS.

\section{Results}

\subsection{Behavioral results}

Healthy controls and patients with schizophrenia were instructed to indicate via button press whether the actor in the video described a person-related content or an object-related content. Correct responses (percentage correct) and their reaction times were analyzed each with mixed ANOVA [within factors: CONTENT (social vs. non-social) and MODALITY (bimodal vs. gesture vs. speech); between factor: GROUP (control vs. patient)]. Descriptive statistics for each experimental condition for both groups were provided in Table 2.

For accuracy, we observed three main effects despite, but no interaction with GROUP $\left(\mathrm{F}_{(2,66)}=0.83, \mathrm{p}=0.44, \eta^{2} \mathrm{G}=0.003\right)$ : Patients were significantly less accurate than healthy controls $\left(\mathrm{F}_{(1,33)}=5.18, \mathrm{p}=\right.$ $\left..03, \eta^{2} G=0.05\right)$. Non-social videos were judged more accurately than social videos $\left(\mathrm{F}_{(1,33)}=19.75, \mathrm{p}=.0001, \eta^{2} \mathrm{G}=0.18\right)$. Additionally, accuracy between the three modalities was different $\left(\mathrm{F}_{(2,66)}=14.53\right.$, $\mathrm{p}$ $=.00001, \eta^{2} G=0.04$ ). Post-hoc pairwise $t$-test showed that the accuracy for the bimodal conditions were highest (vs. gesture, $\mathrm{t}=5.81, \mathrm{p}=$ .000001; vs. speech, $\mathrm{t}=2.77, \mathrm{p}=.007$ ). The accuracy for the speech conditions was also higher than the gesture conditions $(\mathrm{t}=2.14, \mathrm{p}=$ .035).

For reaction times, there were also three main effects, but no interaction with GROUP $\left(\mathrm{F}_{(2,66)}=0.75, \mathrm{p}=0.47, \eta^{2} \mathrm{G}=0.003\right)$. Patients were generally slower than healthy controls $\left(\mathrm{F}_{(1,33)}=19.64, \mathrm{p}=.00001\right.$, $\left.\eta^{2} \mathrm{G}=0.35\right)$. Reaction times for non-social content were faster than social content $\left(F_{(1,33)}=8.63, p=.005, \eta^{2} G=0.005\right)$. Additionally, reaction times for the three modalities were different $\left(F_{(2,66)}=10.14, p\right.$

Table 2

Accuracy and reaction times (RT) for the behavioral task.

\begin{tabular}{|c|c|c|c|c|}
\hline \multicolumn{5}{|l|}{ Accuracy (\%) } \\
\hline \multirow[t]{2}{*}{ Conditions } & \multicolumn{2}{|c|}{ Controls $(n=18)$} & \multicolumn{2}{|c|}{ Patients $(n=17)$} \\
\hline & Mean & SD & Mean & SD \\
\hline Social Bimodal (SB) & 81.84 & 10.78 & 71.79 & 19.99 \\
\hline Social Gesture (SG) & 70.29 & 16.36 & 63.34 & 18.45 \\
\hline Social Speech (SS) & 79.91 & 17.17 & 71.71 & 20.57 \\
\hline Non-social Bimodal (NB) & 93.95 & 6.19 & 91.18 & 12.96 \\
\hline Non-social Gesture (NG) & 89.1 & 10.49 & 82.8 & 11.62 \\
\hline Non-social Speech (NS) & 88.46 & 9.69 & 80.31 & 22.95 \\
\hline \multicolumn{5}{|l|}{ Reaction times (ms) } \\
\hline \multirow[t]{2}{*}{ Conditions } & \multicolumn{2}{|c|}{ Controls $(n=18)$} & \multicolumn{2}{|c|}{ Patients $(n=17)$} \\
\hline & Mean & SD & Mean & SD \\
\hline Social Bimodal (SB) & 3270.26 & 262.57 & 4196.31 & 831.15 \\
\hline Social Gesture (SG) & 3154.67 & 281.27 & 4042.41 & 897.51 \\
\hline Social Speech (SS) & 3455.9 & 279.19 & 4238.1 & 785.66 \\
\hline Non-social Bimodal (NB) & 2982.74 & 262.79 & 4092.35 & 907.69 \\
\hline Non-social Gesture (NG) & 3185.07 & 347.14 & 4122.33 & 924.68 \\
\hline Non-social Speech (NS) & 3244.25 & 325.07 & 4177.06 & 921.39 \\
\hline
\end{tabular}

$\mathrm{SD}$, standard deviation; Reaction times were measured in reference to each video onset (full video length: $5 \mathrm{~s}$ ). 
$\left.=.0001, \eta^{2} \mathrm{G}=0.01\right)$. Post-hoc pairwise $t$-test showed that the reaction time for gesture modality was significantly slower than the other modalities $\left(|t|_{\min }=4.03, p_{\max }=0.0001\right)$, and there was no significant difference between the bimodal and the speech modalities $(\mathrm{t}=0.21, \mathrm{p}=$ .82).

The results from the behavioral task showed that patients with schizophrenia were generally slower and less accurate in the content judgement task. Additionally, the responses for non-social videos were faster and more accurate. With regard to modality, responses in the bimodal conditions were the most accurate and the fastest. However, it has to be noted that, due to the limited sample size (see Discussion), we refrain from elaborating on the interpretation of the observed behavioral effects.

\section{2. $f M R I$ results}

\subsubsection{Social-abstract $(S)>$ Non-social-concrete $(N)$}

We report whole-brain fMRI results for $\mathrm{S}>\mathrm{N}$ comparisons in Fig. 2 and Table 3. For the speech conditions (SS $>$ NS), healthy controls activated an extensive fronto-temporal-parietal network including the bilateral inferior frontal gyrus (IFG) and the temporal lobe, the dorsolateral prefrontal cortex (dlPFC) and MPFC, and the left supramarginal gyrus; patients revealed similar regions for this comparison, and we observed no group difference for social $>$ non-social speech. For the gesture conditions (SG > NG), controls activated the bilateral PFC and IFG; patients activated the bilateral prefrontal cortex. Group interaction (Control (SG > NG) > Patient (SG > NG)) suggests that patients showed reduced activation in the MPFC and the anterior cingulate cortex for the social gesture condition when compared to controls (Fig. 2B). In the bimodal condition ( $\mathrm{SB}>\mathrm{NB}$ ), both controls and patients activated regions similar to that of the speech condition. For patients, we additionally reported modality* content interaction in the Supplement S3, which shows that patients' aberrant processing of social gestures is improved in the bimodal modality.

\subsubsection{Non-social-concrete $(N)>$ Social-abstract $(S)$}

We report whole-brain fMRI results for $\mathrm{N}>\mathrm{S}$ comparisons in Fig. 3 and Table 4.. For the speech conditions (NS > SS), healthy controls activated the left pre/postcentral gyrus, supramarginal gyrus, and the left insula, whereas patients did not reveal any significant activations for this comparison. The group interaction (Control (NS $>$ SS) $>$ Patient
(NS > SS)) suggests that, when compared to controls, patients showed reduced activation in the left postcentral gyrus and the right insula for the processing of non-social content in the speech-only modality (see Fig. 3B). For gesture conditions (NG > SG), controls showed increased activation for the non-social content in the bilateral posterior temporal gyrus, supramarginal gyrus, and occipital cortices, as well as the left pre/postcentral gyrus and the left insula. Patients also activated the bilateral posterior temporal gyrus and occipital lobe, as well as the left pre/postcentral gyrus. The group interaction revealed no significant clusters. For bimodal conditions (NB $>$ SB), both controls and patients activated regions that are comparable to that of the gesture conditions. Additionally, for patients, bimodal input seems to enhance their aberrant processing of non-social speech, as reported in Supplement S3.

\subsection{Exploratory correlation analyses}

In patients, for the NS condition, we found that the accuracy for the NS condition correlate negatively with the SANS composite scores of the patients $(\mathrm{r}=-0.52, \mathrm{p}=0.03$, power $=0.63$; Fig. $4 \mathrm{~A})$. Additionally, SANS 1 (flat affect) and SANS 2 (alogia) scores correlate negatively with the accuracy for the NS condition (SANS $1: \mathrm{r}=-0.62, \mathrm{p}=0.008$, power $=$ 0.79 , Fig. 4B; SANS 2 : $r=-0.63, p=0.006$, power $=0.82$, Fig. $4 \mathrm{C}$ ).

\section{Discussion}

With an fMRI study on the perception of social-abstract and nonsocial-concrete stimuli in auditory-speech, visuo-gestural, and bimodal modalities, we observed modality-specific neural modulation in schizophrenia: In comparison to controls, reduced neural activity in patients was observed only for social gesture and non-social speech. Moreover, in the bimodal condition, neural activation for both social and non-social contents was comparable in patients and controls.

\subsection{Social information processing in schizophrenia}

In the current study, patients showed dissociable neural modulation during the processing of social content in speech and gesture modalities. In the speech modality, both controls and patients activated a leftlateralized set of brain regions, including the dlPFC, $\mathrm{mPFC}$, the IFG, the temporal lobe, and the angular/supramarginal gyrus, without any group difference. This finding replicates results from our previous study

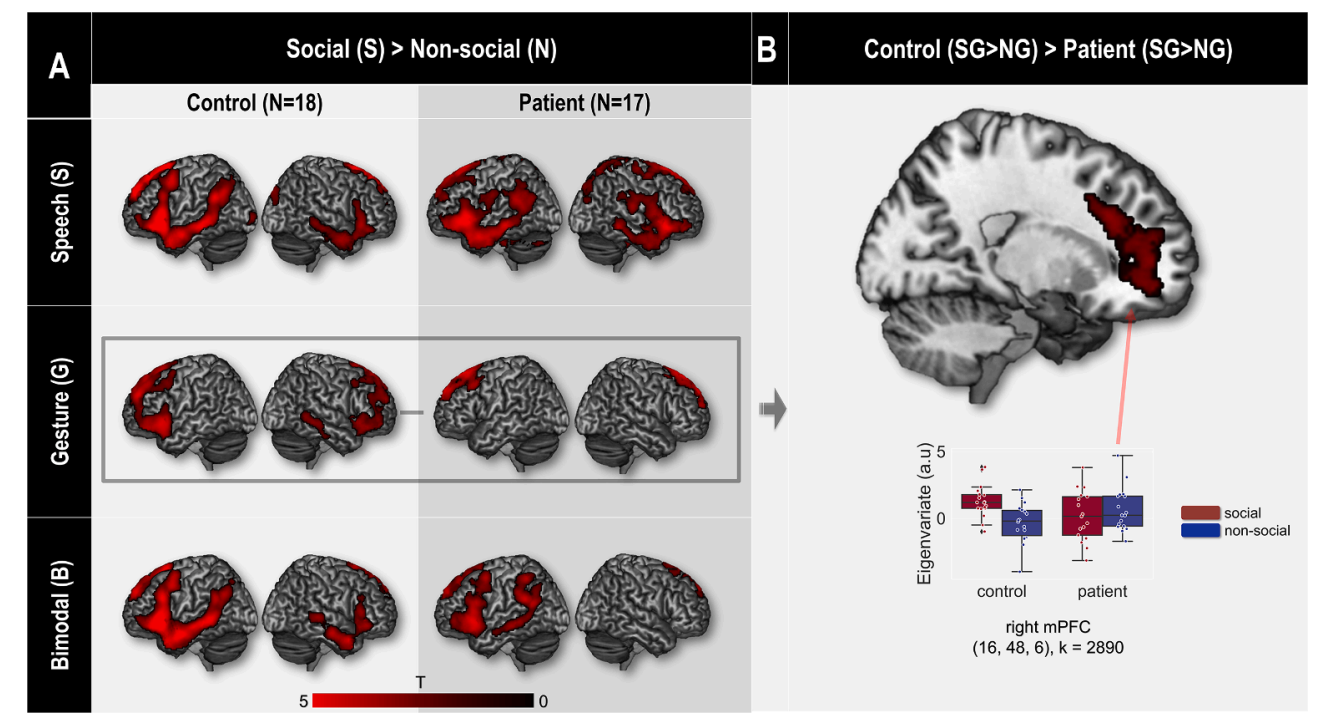

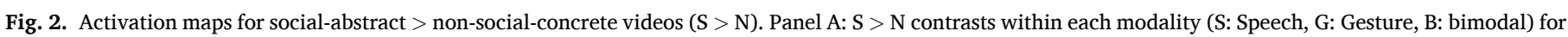

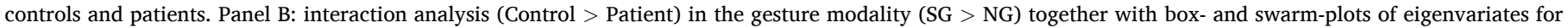
selected clusters. All results are correct for multiple comparison with Monte-Carlo simulation with k $>2268$ voxels. 
Table 3

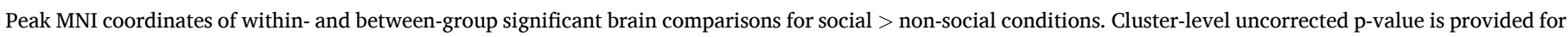
each cluster, asterisk indicates significance $(\mathrm{p}<0.05)$ with cluster-level FWE correction.

\begin{tabular}{|c|c|c|c|c|c|c|c|c|}
\hline \multirow[b]{2}{*}{ Contrasts } & \multirow[b]{2}{*}{ Anatomical Region (Peak) } & \multirow[b]{2}{*}{ Hem. } & \multirow[b]{2}{*}{$\mathrm{k}$} & \multicolumn{3}{|c|}{ Peak MNI coordinates } & \multirow[b]{2}{*}{$\mathrm{t}$} & \multirow[b]{2}{*}{$\mathrm{p}$ (unc.) } \\
\hline & & & & $\mathrm{x}$ & $\mathrm{y}$ & $\mathrm{z}$ & & \\
\hline \multicolumn{9}{|l|}{ Control } \\
\hline \multirow[t]{3}{*}{ SS $>$ NS } & Inferior frontal gyrus pars orbitalis & $\mathrm{L}$ & 15,202 & -48 & 22 & -6 & 6.74 & $<0.001^{*}$ \\
\hline & Middle temporal gyrus & $\mathrm{R}$ & 2816 & 48 & -22 & -14 & 4.05 & 0.008 \\
\hline & Precuneus & $\mathrm{L}$ & 4169 & -4 & -48 & 42 & 3.18 & 0.002 \\
\hline $\mathrm{SG}>\mathrm{NG}$ & Superior frontal gyrus & $\mathrm{L}$ & 16,869 & -22 & 52 & 32 & 4.68 & $<0.001 *$ \\
\hline \multirow[t]{3}{*}{$\mathrm{SB}>\mathrm{NB}$} & Inferior frontal gyrus pars orbitalis & $\mathrm{L}$ & 9637 & -46 & 26 & -4 & 7.05 & $<0.001^{*}$ \\
\hline & Supplementary motor area & $\mathrm{L}$ & 4916 & -8 & 16 & 66 & 5.3 & $0.001 *$ \\
\hline & Middle temporal pole & $\mathrm{R}$ & 3023 & 50 & 10 & -28 & 4.64 & 0.006 \\
\hline \multicolumn{9}{|l|}{ Patient } \\
\hline \multirow[t]{2}{*}{ SS $>$ NS } & Inferior frontal gyrus pars orbitalis & $\mathrm{L}$ & 33,204 & -50 & 20 & -4 & 5.77 & $<0.001 *$ \\
\hline & Supplementary motor area & $\mathrm{L}$ & 7862 & -2 & 24 & 68 & 4.39 & $<0.001^{*}$ \\
\hline $\mathrm{SG}>\mathrm{NG}$ & Superior frontal gyrus & $\mathrm{L}$ & 2836 & -16 & 32 & 62 & 3.86 & 0.008 \\
\hline \multirow[t]{3}{*}{$\mathrm{SB}>\mathrm{NB}$} & Inferior frontal gyrus pars triangularis & $\mathrm{L}$ & 4577 & -50 & 24 & -2 & 4.83 & 0.001 \\
\hline & Supramarginal gyrus & $\mathrm{L}$ & 3094 & -58 & -46 & 26 & 4.33 & 0.006 \\
\hline & Medial superior frontal gyrus & $\mathrm{L}$ & 3155 & -4 & 28 & 44 & 4.03 & 0.005 \\
\hline \multicolumn{9}{|c|}{ Interaction: Control > Patient } \\
\hline $\mathrm{SS}>\mathrm{NS}$ & N. $S$. & & & & & & & \\
\hline \multirow[t]{2}{*}{$\mathrm{SG}>\mathrm{NG}$} & Anterior cingulate cortex & $\mathrm{R}$ & 2890 & 16 & 30 & 24 & 3.04 & 0.007 \\
\hline & Medial superior frontal gyrus & $\mathrm{R}$ & & 16 & 48 & 6 & 2.95 & \\
\hline $\mathrm{SB}>\mathrm{NB}$ & N. S. & & & & & & & \\
\hline
\end{tabular}

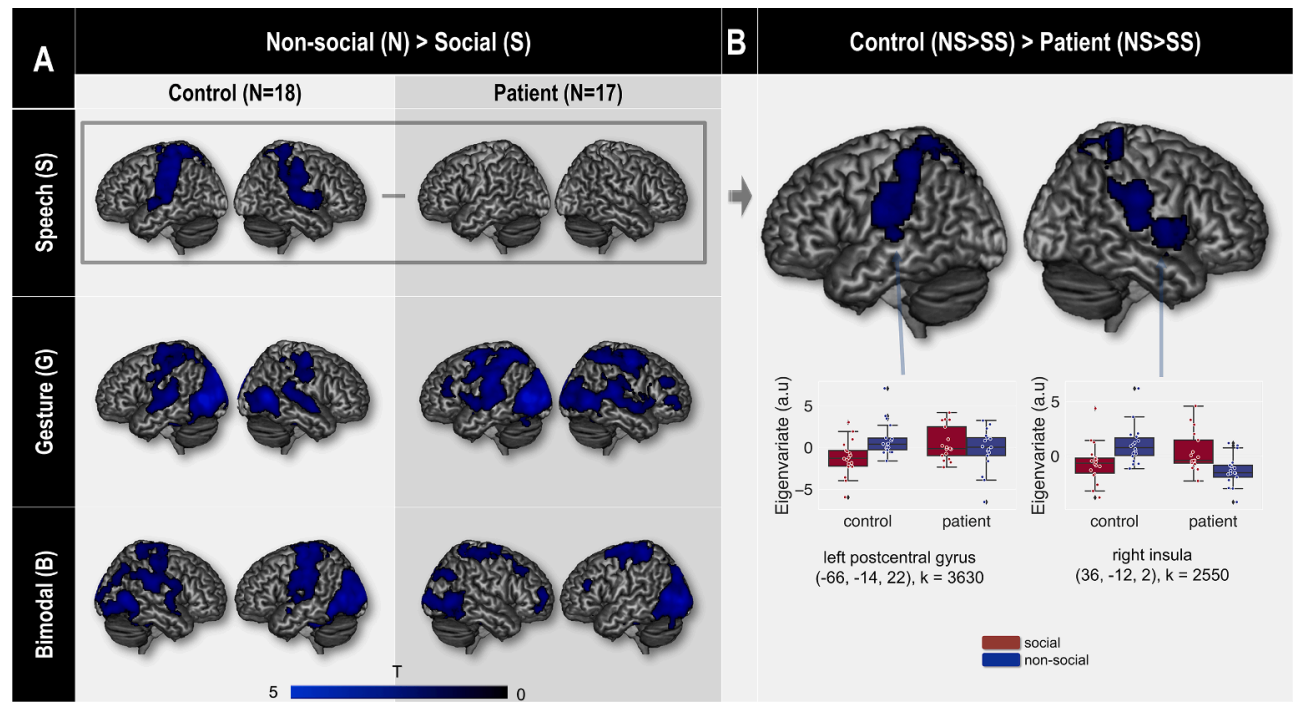

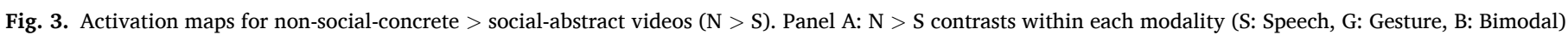

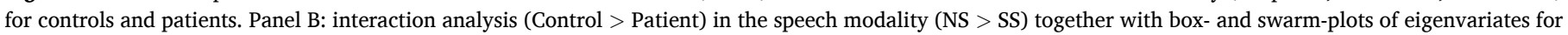
selected clusters. All results are correct for multiple comparison with Monte-Carlo simulation with $\mathrm{k}>2268$ voxels.

showing supramodal social-abstract processing of healthy individuals (Straube et al., 2013c), and is consistent with earlier studies in basic research on the role of the MPFC in both perceiving social-related stimuli and mentalizing social intentions (Mitchell et al., 2005, 2002; Van Overwalle, 2009). The observed left IFG and temporal lobe activation is also in line with the literature on the neural substrates of abstract vs. concrete semantics (Binder et al., 2009, 2005), as the videos in the social-abstract condition, irrespective of modality, are more abstract than the non-social, object-related condition. The fact that we did not find any group differences in social-abstract speech processing suggests that patients with schizophrenia exhibit intact neural processing of social content presented in this modality. This finding complies with a previous language study in schizophrenia, in which patients also activated a comparable left fronto-temporal network to controls when they processed abstract vs. concrete visual sentences (Kuperberg et al., 2008). Together, although schizophrenia is well-known for its social cognition deficits (Green et al., 2015), as well as impairments in the perception of affective face or pitch (Edwards et al., 2001; Leitman et al., 2005; Morris et al., 2009), patient's processing of social speech may remain intact. In the gesture modality, however, although patients activated the mPFC for the social vs. non-social stimuli, this activation was reduced when compared to controls. Notably, such modality-specific neural modulation is, for the first time, reported for social information processing in schizophrenia. Notably, in addition to the mPFC modulation, in schizophrenia, we did not observe the activation for social gestures in the left IFG. The left IFG has been linked to motor planning of gesture (Walther et al., 2013); and, together with the DLPFC, left IFG activation has been shown to be modulated in schizophrenia for social gesture planning (Stegmayer et al., 2018). In addition to the left IFG, the left middle temporal gyrus (MTG) activation was not observed for patients with schizophrenia when processing social gestures. Importantly, functional and structural aberrance in both the left IFG and MTG are highly indicative of major language deficits (e.g., formal thought disorders) in schizophrenia (Kircher et al., 2018; Wensing et al., 2017). Here, 
Table 4

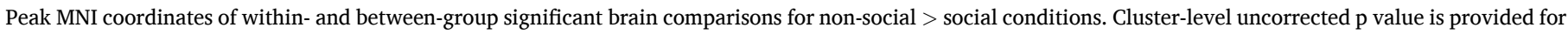
each cluster, asterisk indicates signifance $(\mathrm{p}<0.05)$ with cluster-level FWE correction.

\begin{tabular}{|c|c|c|c|c|c|c|c|c|}
\hline \multirow[b]{2}{*}{ Contrasts } & \multirow[b]{2}{*}{ Anatomical Region (Peak) } & \multirow[b]{2}{*}{ Hem. } & \multirow[b]{2}{*}{$\mathrm{k}$} & \multicolumn{3}{|c|}{ Peak MNI coordinates } & \multirow[b]{2}{*}{$\mathrm{t}$} & \multirow[b]{2}{*}{$\mathrm{p}$ (unc.) } \\
\hline & & & & $\mathrm{x}$ & $\mathrm{y}$ & $\mathrm{z}$ & & \\
\hline \multicolumn{9}{|l|}{ Control } \\
\hline NS $>$ SS & Middle cingulate cortex & $\mathrm{L}$ & 17,667 & -48 & -28 & 42 & 4.18 & $<0.001 *$ \\
\hline \multirow[t]{2}{*}{$\mathrm{NG}>\mathrm{SG}$} & Middle Occipital gyrus & $\mathrm{L}$ & 18,822 & -46 & -72 & 4 & 6.48 & $<0.001^{*}$ \\
\hline & Middle temporal gyrus & $\mathrm{R}$ & 2620 & 50 & -64 & 8 & 5.01 & 0.01 \\
\hline \multirow[t]{2}{*}{$\mathrm{NB}>\mathrm{SB}$} & Middle temporal gyrus & $\mathrm{L}$ & 15,424 & -50 & -72 & 6 & 4.55 & $<0.001^{*}$ \\
\hline & Supplementary motor area & $\mathrm{R}$ & 8910 & 6 & -12 & 56 & 3.7 & $<0.001 *$ \\
\hline \multicolumn{9}{|l|}{ Patient } \\
\hline $\mathrm{NS}>\mathrm{SS}$ & N. S. & & & & & & & \\
\hline $\mathrm{NG}>\mathrm{SG}$ & Middle temporal gyrus & $\mathrm{L}$ & 49,718 & -48 & -70 & 6 & 6.9 & $<0.001^{*}$ \\
\hline \multirow[t]{2}{*}{$\mathrm{NB}>\mathrm{SB}$} & Middle occipital gyrus & $\mathrm{L}$ & 22,719 & -30 & -88 & 2 & 4.86 & $<0.001^{*}$ \\
\hline & Olfactory cortex & $\mathrm{L}$ & 3347 & -8 & 26 & -4 & 4.2 & 0.004 \\
\hline \multicolumn{9}{|c|}{ Interaction: Control > Patient } \\
\hline \multirow[t]{2}{*}{$\mathrm{NS}>\mathrm{SS}$} & Insula & $\mathrm{R}$ & 2550 & 36 & -12 & 2 & 3.05 & 0.011 \\
\hline & Postcentral gyrus & $\mathrm{L}$ & 3630 & -66 & -14 & 22 & 2.92 & 0.003 \\
\hline $\mathrm{NG}>\mathrm{SG}$ & N. S. & & & & & & & \\
\hline $\mathrm{NB}>\mathrm{SB}$ & N. S. & & & & & & & \\
\hline
\end{tabular}

A

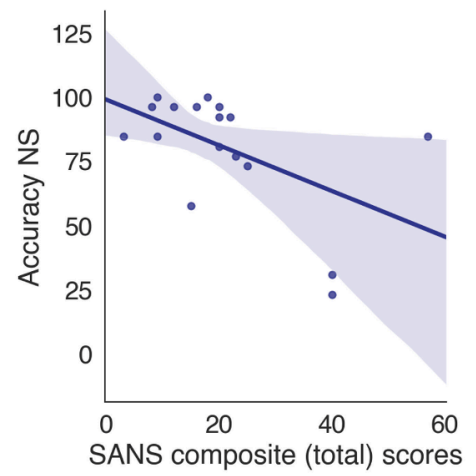

B

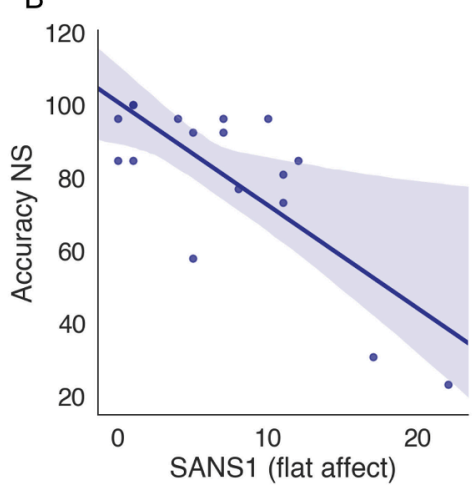

C

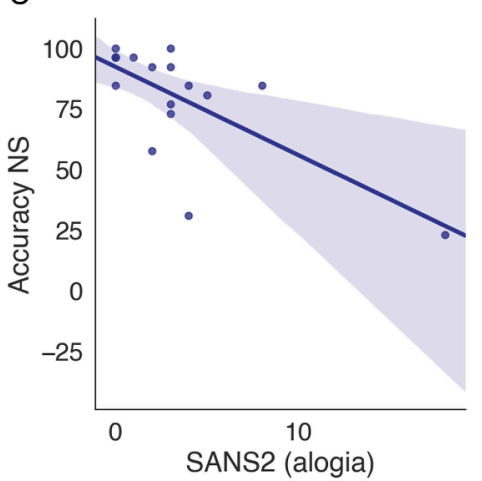

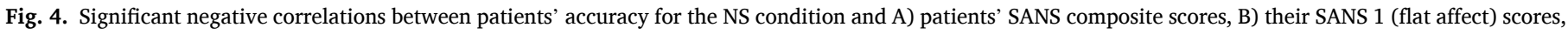
and C) their SANS 2 (alogia) scores.

however, rather than showing a modality (speech) -specific neural modulation, our results indicate that these regions indicate a crossmodality deficit, and echo prior research on potential neural aberrance in these regions regarding integration between speech and gesture at both lower-perceptual and higher-semantic levels (Straube et al., 2013a; Wroblewski et al., 2020). To summarize, our results, from a processing perspective, corroborate these extant studies, showing that functional modulation in the PFC, and potentially in the left IFG and MTG, may give rise to gesture-specific social perception deficits.

\subsection{Non-social information processing in schizophrenia}

For the processing of non-social (object-related) information, again, the neural modulation in patients showed an apparent dissociation. In the gesture modality, both controls and patients activated the bilateral occipital-parietal cortices, STG, LOTC, insula, and the pre/postcentral gyrus. In the speech modality, although these regions were similarly activated in controls, their brain activation was significantly reduced for patients. The group comparison suggests reduced activation in schizophrenia patients in the bilateral insula and the left postcentral gyrus for non-social speech. Of note, the observed regions for non-social and object-related information processing overlap with regions considered as part of the putative mirror neuron network, which is not only important for action observation and imitation, but also for the understanding of object- and motor-related features in verbal form (Buccino et al., 2001; Di Pellegrino et al., 1992; Iacoboni et al., 1999; Rizzolatti and Craighero, 2004). This process would require mental simulation of sensorimotor experience (Barsalou, 2008; Pulvermüller, 2005; Pulvermüller et al., 2005). Additionally, the LOTC is also crucially involved in the perception of biological motion, object, face, as well as tool-use (Bi et al., 2016; Goodale and Milner, 1992; Higuchi et al., 2007; Lingnau and Downing, 2015; Nickl-Jockschat et al., 2015). Moreover, these regions are also reported to support the processing of concrete linguistic information (Binder et al., 2009, 2005). Our data from the control group suggest that these regions support the processing of non-social-concrete features, irrespective of encoding modality. This finding is in line with the embodiment view of action and language processing (Barsalou, 2008; De Stefani and De Marco, 2019). With regard to the patients, we observed normal neural processing of non-social content in the gesture modality, supporting a previous study (Horan et al., 2014), which reported intact mirror neuron activity in schizophrenia (but see (Thakkar et al., 2014)). However, as we also observed reduced bilateral postcentral gyrus and right insula activity for patients for non-social speech, in turn, this would imply that motor simulation, as required for processing object-related features from auditory speech, might still be impaired in schizophrenia (Kuperberg et al., 2008; Thakkar et al., 2014). This impairment concurs with the reported deficits of schizophrenia in action imitation (Matthews et al., 2013; Mehta et al., 2014; Park et al., 2008; Thakkar et al., 2014; Walther et al., 2015, 2013), where certain degrees of motor simulation is required. However, adding to prior research on schizophrenia's motor impairments, our results provide a more nuanced version: Motor-related semantics as delivered by pantomime does not necessarily lead to reduced neural activation in schizophrenia; rather, modulated neural activity is only observed for speech 
contents simulating these concrete motor-actions. Additionally, schizophrenia is commonly related to structural changes in the left insula; this region is also functionally relevant to a range of language and speech processing aberrances in schizophrenia (Nickl-Jockschat et al., 2011; Ruiz et al., 2013; White et al., 2010). Therefore, functional abnormality in this region might also contribute to the simulation of motor events with speech. In sum, our fMRI results may suggest that patients with schizophrenia are impaired in a mirror-mechanism simulating concretemotor events with speech.

In the NS condition, we also observed negative correlation between patients' SANS composite and subscores and their task accuracy. This evidence converges with previous research, corroborating the potential role of the mirror neuron system during embodiment of non-social information (e.g., action imitation and observation), as well as its relation to the development and persistence of negative symptoms (Buccino and Amore, 2008; Mehta et al., 2014). Our correlational finding, although being exploratory (see 4.4), links the mirror neuron system to the reported studies showing correlation between compromised gesture performance and more severe negative symptoms (Park et al., 2008; Walther et al., 2019), tentatively suggests that the theoretical link between gesture and negative symptom may partially derive from motorsimulation. Notably, in the current study, for social processing, we did no observe any correlations between brain activations / behavior and symptom measures, especially positive symptoms such as hallucinations, and positive formal thought disorders, as in Straube et al. (2013a). Clearly, the exact relationship between social/non-social information processing and major symptoms of schizophrenia needs to be addressed by further research with larger samples.

\subsection{Enhancing modality-specific information processing deficits with bimodal input}

The novelty of our findings lies in the dissociable modalityspecificity concerning dysfunctional neural processing of social and non-social features. Social-abstract and non-social-concrete features are functionally and neurally dissociable at the representational level (Binder et al., 2009; Mitchell et al., 2005, 2002). Besides, they might be differentially processed through either linguistic (speech) or nonlinguistic (gesture) channels. It has been proposed that social-abstract concepts may be preferentially represented in speech, and that nonsocial concrete concepts are preferentially delivered in hand action and gesture (Paivio, 2010; Perlovsky and Ilin, 2013). Despite this theoretical proposal, however, during comprehension, healthy participants are able to process both types of information in a supramodal manner (e.g., semantic processing with unitary core systems, irrespective of encoding modality, as in (Pulvermüller et al., 2005; Straube et al., 2013c)). For patients with schizophrenia, as they exhibit similar neural activations when processing social speech and non-social gestures to controls, this might be an indication that they are at least intact in processing these contents through a preferred modality at representational level. But, they might show activation reduction in relevant regions when these features are conveyed in a non-preferred modality, as the processing of these features would require some form of mental simulation: In the case nonsocial information, patients are impaired in the simulation of motorrelated experience from action to language (Pulvermüller et al., 2005); In contrast, when patients are presented with social information, they might be impaired when simulating social features encoded by hand gestures (but not with speech), as shown in their reduced mPFC activation. This observed modality-specific processing deficit might also suggest that patients, unlike controls, are not capable of processing social/non-social information in a supramodal manner like healthy participants, as reported in previous studies (Straube et al., 2012, 2013c). More importantly, extending previous studies on aberrant processing of social/non-social content in schizophrenia, our results indicate that this neural deficit is not universally present for either a specific modality or content, but rather appears only in specific combinations of these two factors.

Despite reduced neural processing of both social and non-social content in gesture and speech modalities, patients displayed intact neural processing of these features, as well as improved task accuracy in the bimodal conditions. This enhancement effect concurs with a line of proposals (Holler and Levinson, 2019), who argue for a bi-directional facilitative relation between speech and gesture (for empirical evidence, see (Cuevas et al., 2019; Drijvers et al., 2018; He et al., 2018b; Krahmer and Swerts, 2007; Wang and Chu, 2013)). More importantly, our finding extends previous basic research, suggesting the translational implication of this mechanism. In schizophrenia research, the past decade has witnessed substantial progress in the development of social cognitive training in schizophrenia (Kurtz et al., 2016; Kurtz and Richardson, 2012), with recent innovation regarding the incorporation of social stimuli from a broader range of modalities (Nahum et al., 2014). Our findings extend these approaches, proposing potential therapeutic implications of deploying naturalistic and multimodal stimuli during social cognitive training (Riedl et al., 2020), as they might be able to normalize processing of both social and non-social information, at least at a neural level. Future research is expected to further explore whether the neural enhancements can be linked to functional outcome after social cognitive training in a multimodal setting.

\subsection{Limitations}

Despite new insights, our study has limitations in several aspects. Firstly, the sample size of this study ( $n=17$ and 18 for each group) falls within the required minimum of this type of studies (Friston, 2013). Hence, the patient group was limited to medicated chronic patients, and we were thus unable to test if the neural pattern is comparable to firstepisode schizophrenia (Kindler et al., 2019), and were unable to attribute the findings to schizophrenia without separating out the effects of medication. Secondly, due to the limited sample size and the exploratory nature, the findings resulted from the correlation analyses needs to be treated with caution. Thirdly, our the reported group interaction did not survive the most conservative FWE threshold. Future replications are thus necessary to validate if the observed effects are replicatable when a larger sample is possible. Lastly, we did not observe any reliable relationship between BOLD signal and behavioral results: More careful, and neurobiologically plausible behavioral tasks on the social/non-social information processing are necessary for future experiments, so that their processing deficits in schizophrenia can be better examined by clinical neuroscientists and practitioners.

\subsection{Conclusion}

Here, in an fMRI study, we for the first time showed modalityspecific neural modulation in schizophrenia when patients process social-abstract and non-social-concrete content in speech and gesture. Moreover, these deficits could be compensated when both speech and gesture were presented together. Our findings provide novel insights on dysfunctional multimodal communication in schizophrenia, and suggest potential therapeutic implications of employing multimodal social cognitive intervention in schizophrenia.

\section{Declaration of Competing Interest}

The authors declare that they have no known competing financial interests or personal relationships that could have appeared to influence the work reported in this paper.

\section{Acknowledgements}

This research project is supported by a grant from the 'Von-BehringRöntgen-Stiftung' (project no. 59-0002 and 64-0001) and by the 'Deutsche Forschungsgemeinschaft' (project no. DFG: STR1146/11-2 \& 
KI588/6-2, HE8029/2-1, and CRC/TRR 135/2 project A3, number: 222641018). The study was also supported by the Core Facility Brain Imaging, Faculty of Medicine, University of Marburg, Rudolf-BultmannStr. 9, 35039, Marburg, Germany. The manuscript has been published as a preprint on bioRxiv.

\section{Appendix A. Supplementary data}

Supplementary data to this article can be found online at https://doi. org/10.1016/j.nicl.2021.102568.

\section{References}

Amodio, D.M., Frith, C.D., 2006. Meeting of minds: the medial frontal cortex and social cognition. Nat. Rev. Neurosci. 7 (4), 268-277.

Andreasen, N. C. (1981). Scale for the Assessment of Negative Symptoms (SANS).

Andreasen, N.C., 1984. Scale for the Assessment of Positive Symptons:(SAPS). University of Iowa.

Anticevic, A., Van Snellenberg, J.X., Cohen, R.E., Repovs, G., Dowd, E.C., Barch, D.M., 2012. Amygdala recruitment in schizophrenia in response to aversive emotional material: a meta-analysis of neuroimaging studies. Schizophr. Bull. 38 (3), 608-621. Barsalou, L.W., 2008. Grounded Cognition. Annu. Rev. Psychol. 59 (1), 617-645.

Barth, A., Küfferle, B., 2001. Development of a proverb test for assessment of concrete thinking problems in schizophrenic patients. Der Nervenarzt 72 (11), 853-858.

Bi, Y., Wang, X., Caramazza, A., 2016. Object domain and modality in the ventral visual pathway. Trends Cogn. Sci. 20 (4), 282-290.

Binder, J.R., Desai, R.H., Graves, W.W., Conant, L.L., 2009. Where is the semantic system? A critical review and meta-analysis of 120 functional neuroimaging studies. Cerebral Cortex 19 (12), 2767-2796.

Binder, J.R., Westbury, C.F., McKiernan, K.A., Possing, E.T., Medler, D.A., 2005. Distinct Brain Systems for Processing Concrete and Abstract Concepts. J. Cognit. Neurosci. 17 (6), 905-917.

Bleuler, E. (1950). Dementia praecox or the group of schizophrenias.

Brown, M., Kuperberg, G.R., 2015. A Hierarchical Generative Framework of Language Processing: Linking Language Perception, Interpretation, and Production Abnormalities in Schizophrenia. Front. Hum. Neurosci. 9 (e29315), 611. https://doi. org $/ 10.3389 /$ fnhum.2015.00643.

Buccino, G., Amore, M., 2008. Mirror neurons and the understanding of behavioural symptoms in psychiatric disorders. Curr. Opin. Psych. 21 (3), 281-285.

Buccino, G., Binkofski, F., Fink, G.R., Fadiga, L., Fogassi, L., Gallese, V., Seitz, R.J., Zilles, K., Rizzolatti, G., Freund, H.-J., 2001. Action observation activates premotor and parietal areas in a somatotopic manner: an fMRI study. Eur. J. Neurosci. 13 (2), 400-404.

Chao, L.L., Haxby, J.V., Martin, A., 1999. Attribute-based neural substrates in temporal cortex for perceiving and knowing about objects. Nat. Neurosci. 2 (10), 913-919.

Cuevas, P., Steines, M., He, Y., Nagels, A., Culham, J., Straube, B., 2019. The facilitative effect of gestures on the neural processing of semantic complexity in a continuous narrative. NeuroImage 195, 38-47.

De Stefani, E., De Marco, D., 2019. Language, Gesture, and Emotional Communication: An Embodied View of Social Interaction. Front. Psychol. 10.

di Pellegrino, G., Fadiga, L., Fogassi, L., Gallese, V., Rizzolatti, G., 1992. Understanding motor events: a neurophysiological study. Exp. Brain Res. 91 (1), 176-180.

Drijvers, L., Özyürek, A., Jensen, O., 2018. Hearing and seeing meaning in noise: Alpha, beta, and gamma oscillations predict gestural enhancement of degraded speech comprehension. Hum. Brain Mapp. 39 (5), 2075-2087.

Edwards, J., Pattison, P.E., Jackson, H.J., Wales, R.J., 2001. Facial affect and affective prosody recognition in first-episode schizophrenia. Schizophr. Res. 48 (2-3), 235-253.

Friedman, T., Sehatpour, P., Dias, E., Perrin, M., Javitt, D.C., 2012. Differential Relationships of Mismatch Negativity and Visual P1 Deficits to Premorbid Characteristics and Functional Outcome in Schizophrenia. Biol. Psychiatry 71 (6), 521-529.

Friston, K., 2013. Sample size and the fallacies of classical inference. NeuroImage 81, 503-504.

FRITH, C.D., 2004. Schizophrenia and theory of mind. Psychol. Med. 34 (3), 385-389.

Frith, C.D., Frith, U., 2006. The neural basis of mentalizing. Neuron 50 (4), 531-534.

Goodale, M.A., Milner, A.D., 1992. Separate visual pathways for perception and action. Trends Neurosci. 15 (1), 20-25.

Green, M.F., Horan, W.P., Lee, J., 2015. Social cognition in schizophrenia. Nat. Rev. Neurosci. 16 (10), 620-631.

Gur, R.E., McGrath, C., Chan, R.M., Schroeder, L., Turner, T., Turetsky, B.I., Kohler, C., Alsop, D., Maldjian, J., Ragland, J.D., Gur, R.C., 2002. An fMRI Study of Facial Emotion Processing in Patients With Schizophrenia. Am. J. Psychiatry 159 (12), 1992-1999.

He, Y., Gebhardt, H., Steines, M., Sammer, G., Kircher, T., Nagels, A., Straube, B., 2015. The EEG and fMRI signatures of neural integration: An investigation of meaningful gestures and corresponding speech. Neuropsychologia 72, 27-42.

He, Y., Luell, S., Muralikrishnan, R., Straube, B., Nagels, A., 2020. Gesture's body orientation modulates the N400 for visual sentences primed by gestures. Hum. Brain Mapp.
He, Y., Nagels, A., Schlesewsky, M., Straube, B., 2018a. The role of gamma oscillations during integration of metaphoric gestures and abstract speech. Front. Psychol. 9, 1348. https://doi.org/10.3389/fpsyg.2018.01348.

He, Y., Steines, M., Sammer, G., Nagels, A., Kircher, T., Straube, B., 2018b. ActionRelated Speech Modulates Beta Oscillations During Observation of Tool-Use Gestures. Brain Topogr. 31 (5), 838-847.

He, Y., Steines, M., Sommer, J., Gebhardt, H., Nagels, A., Sammer, G., Kircher, T., Straube, B., 2018c. Spatial-temporal dynamics of gesture-speech integration: a simultaneous EEG-fMRI study. Brain Struct. Funct. 223 (7), 3073-3089.

Higuchi, S., Imamizu, H., Kawato, M., 2007. Cerebellar Activity Evoked By Common Tool-Use Execution And Imagery Tasks: An Fmri Study. Cortex 43 (3), 350-358.

Holler, J., Levinson, S.C., 2019. Multimodal Language Processing in Human Communication. Trends Cogn. Sci. 23 (8), 639-652.

Horan, W.P., Iacoboni, M., Cross, K.A., Korb, A., Lee, J., Nori, P., Quintana, J., Wynn, J. K., Green, M.F., 2014. Self-reported empathy and neural activity during action imitation and observation in schizophrenia. NeuroImage: Clinical 5, 100-108.

Iacoboni, M., Woods, R.P., Brass, M., Bekkering, H., Mazziotta, J.C., Rizzolatti, G., 1999. Cortical mechanisms of human imitation. Science 286 (5449), 2526-2528.

Järveläinen, J., Schürmann, M., Hari, R., 2004. Activation of the human primary motor cortex during observation of tool use. NeuroImage 23 (1), 187-192.

Johnson-Frey, S.H., 2004. The neural bases of complex tool use in humans. Trends Cogn. Sci. 8 (2), 71-78.

Johnson-Frey, S.H., Maloof, F.R., Newman-Norlund, R., Farrer, C., Inati, S., Grafton, S.T., 2003. Actions or Hand-Object Interactions? Human Inferior Frontal Cortex and Action Observation. Neuron 39 (6), 1053-1058.

Kindler, J., Michel, C., Schultze-Lutter, F., Felber, G., Hauf, M., Schimmelmann, B.G., Kaess, M., Hubl, D., Walther, S., 2019. Functional and structural correlates of abnormal involuntary movements in psychosis risk and first episode psychosis. Schizophr. Res. 212, 196-203.

Kircher, T., Bröhl, H., Meier, F., Engelen, J., 2018. Formal thought disorders: from phenomenology to neurobiology. The Lancet Psychiatry 5 (6), 515-526.

KIRCHER, T.T.J., LIDDLE, P.F., BRAMMER, M.J., WILLIAMS, S.C.R., MURRAY, R.M., McGUIRE, P.K., 2002. Reversed lateralization of temporal activation during speech production in thought disordered patients with schizophrenia. Psychol. Med. 32 (3), 439-449.

Krahmer, E., Swerts, M., 2007. The effects of visual beats on prosodic prominence: Acoustic analyses, auditory perception and visual perception. J. Mem. Lang. 57 (3), 396-414.

Kuperberg, G.R., West, W.C., Lakshmanan, B.M., Goff, D., 2008. Functional Magnetic Resonance Imaging Reveals Neuroanatomical Dissociations During Semantic Integration in Schizophrenia. Biol. Psychiatry 64 (5), 407-418.

Kurtz, M.M., Gagen, E., Rocha, N.B.F., Machado, S., Penn, D.L., 2016. Comprehensive treatments for social cognitive deficits in schizophrenia: A critical review and effectsize analysis of controlled studies. Clinical Psychology Review 43, 80-89.

Kurtz, M.M., Richardson, C.L., 2012. Social Cognitive Training for Schizophrenia: A Meta-Analytic Investigation of Controlled Research. Schizophr. Bull. 38 (5), 1092-1104.

Lehrl, S. (1999). Mehrfachwahl-Wortschatz-Intelligenztest: MWT-B: Spitta.

Leitman, D.I., Foxe, J.J., Butler, P.D., Saperstein, A., Revheim, N., Javitt, D.C., 2005. Sensory Contributions to Impaired Prosodic Processing in Schizophrenia. Biol Psychiatry 58 (1), 56-61.

Lingnau, A., Downing, P.E., 2015. The lateral occipitotemporal cortex in action. Trends Cogn. Sci. 19 (5), 268-277.

Matthews, N., Gold, B.J., Sekuler, R., Park, S., 2013. Gesture imitation in schizophrenia, Schizophr. Bull. 39 (1), 94-101.

Mehta, U.M., Thirthalli, J., Aneelraj, D., Jadhav, P., Gangadhar, B.N., Keshavan, M.S., 2014. Mirror neuron dysfunction in schizophrenia and its functional implications: A systematic review. Schizophr. Res. 160 (1-3), 9-19.

Meyer, L., Lakatos, P., \& He, Y. (2020). Language Dysfunction in Schizophrenia: Assessing Neural Tracking to Characterize the Underlying Disorder (s)? PsyArXiv.

Millman, Z.B., Goss, J., Schiffman, J., Mejias, J., Gupta, T., Mittal, V.A., 2014. Mismatch and lexical retrieval gestures are associated with visual information processing, verbal production, and symptomatology in youth at high risk for psychosis. Schizophr. Res. 158 (1-3), 64-68.

Mitchell, J.P., Banaji, M.R., Macrae, C.N., 2005. General and specific contributions of the medial prefrontal cortex to knowledge about mental states. NeuroImage 28 (4), $757-762$.

Mitchell, J.P., Heatherton, T.F., Macrae, C.N., 2002. Distinct neural systems subserve person and object knowledge. Proc. Natl. Acad. Sci. 99 (23), 15238-15243.

Mittal, V. A., Tessner, K. D., McMillan, A. L., Delawalla, Z., Trotman, H. D., \& Walker, E. F. (2006). Gesture behavior in unmedicated schizotypal adolescents. Journal of Abnormal Psychology, 115(2), 351.

Morris, R.W., Weickert, C.S., Loughland, C.M., 2009. Emotional face processing in schizophrenia: Current Opinion in Psychiatry 22 (2), 140-146.

Nagels, A., Kircher, T., Grosvald, M., Steines, M., Straube, B., 2019. Evidence for gesturespeech mismatch detection impairments in schizophrenia. Psychiatry Res. 273, 15-21.

Nagels, A., Kircher, T., Steines, M., Grosvald, M., Straube, B., 2015a. A brief self-rating scale for the assessment of individual differences in gesture perception and production. Learning and Individual Differences 39, 73-80.

Nagels, A., Kircher, T., Steines, M., Straube, B., 2015b. Feeling addressed! The role of body orientation and co-speech gesture in social communication: Feeling Addressed. Hum. Brain Mapp. 36 (5), 1925-1936.

Nahum, M., Fisher, M., Loewy, R., Poelke, G., Ventura, J., Nuechterlein, K.H., Vinogradov, S., 2014. A novel, online social cognitive training program for young 
adults with schizophrenia: A pilot study. Schizophrenia Research: Cognition 1 (1), e11-e19.

Nickl-Jockschat, T., Rottschy, C., Thommes, J., Schneider, F., Laird, A.R., Fox, P.T., Eickhoff, S.B., 2015. Neural networks related to dysfunctional face processing in autism spectrum disorder. Brain Struct. Funct. 220 (4), 2355-2371.

Nickl-Jockschat, T., Schneider, F., Pagel, A.D., Laird, A.R., Fox, P.T., Eickhoff, S.B., 2011. Progressive pathology is functionally linked to the domains of language and emotion: meta-analysis of brain structure changes in schizophrenia patients. Eur. Arch. Psychiatry Clin. Neurosci. 261 (S2), 166-171.

Oldfield, R.C., 1971. The assessment and analysis of handedness: The Edinburgh inventory. Neuropsychologia 9 (1), 97-113.

Paivio, A., 2010. Dual coding theory and the mental lexicon. The Mental Lexicon 5 (2), 205-230.

Park, S., Matthews, N., Gibson, C., 2008. Imitation, simulation, and schizophrenia. Schizophr. Bull. 34 (4), 698-707.

Perlovsky, L.I., Ilin, R., 2013. Mirror neurons, language, and embodied cognition. Neural Networks 41, 15-22.

Pulvermüller, F., 2005. Brain mechanisms linking language and action. Nat. Rev. Neurosci. 6 (7), 576-582.

Pulvermüller, F., Shtyrov, Y., Ilmoniemi, R., 2005. Brain Signatures of Meaning Access in Action Word Recognition. J. Cognit. Neurosci. 17 (6), 884-892.

Riedl, L., Nagels, A., Sammer, G., Straube, B., 2020. A multimodal speech-gesture training intervention for patients with schizophrenia and its neural underpinnings-the study protocol of a randomized controlled pilot trial. Front. Psychiatry 11, 110.

Rizzolatti, G., Craighero, L., 2004. The mirror-neuron system. Annu. Rev. Neurosci. 27, 169-192.

Ruiz, S., Lee, S., Soekadar, S.R., Caria, A., Veit, R., Kircher, T., Sitaram, R., 2013. Acquired self-control of insula cortex modulates emotion recognition and brain network connectivity in schizophrenia. Hum. Brain Mapp. 34 (1), 200-212.

Saxe, R., Powell, L.J., 2006. It's the thought that counts: specific brain regions for one component of theory of mind. Psychol. Sci. 17 (8), 692-699.

Sergi, M.J., Rassovsky, Y., Widmark, C., Reist, C., Erhart, S., Braff, D.L., Green, M.F., 2007. Social cognition in schizophrenia: relationships with neurocognition and negative symptoms. Schizophr. Res. 90 (1-3), 316-324.

Slotnick, S.D., 2017. Resting-state fMRI data reflects default network activity rather than null data: A defense of commonly employed methods to correct for multiple comparisons. Cognitive neuroscience 8 (3), 141-143.

Slotnick, S.D., Moo, L.R., Segal, J.B., Hart Jr, J., 2003. Distinct prefrontal cortex activity associated with item memory and source memory for visual shapes. Cognitive Brain Research 17 (1), 75-82.

Stegmayer, K., Bohlhalter, S., Vanbellingen, T., Federspiel, A., Wiest, R., Müri, R.M., Walther, S., 2018. Limbic interference during social action planning in schizophrenia. Schizophr. Bull. 44 (2), 359-368.

Straube, B., Green, A., Jansen, A., Chatterjee, A., Kircher, T., 2010. Social cues, mentalizing and the neural processing of speech accompanied by gestures. Neuropsychologia 48 (2), 382-393.

Straube, B., Green, A., Sass, K., Kircher, T., 2013a. Superior temporal sulcus disconnectivity during processing of metaphoric gestures in schizophrenia. Schizophr. Bull. 40 (4), 936-944. https://doi.org/10.1093/schbul/sbt110.

Straube, B., Green, A., Sass, K., Kirner-Veselinovic, A., Kircher, T., 2013b. Neural integration of speech and gesture in schizophrenia: Evidence for differential processing of metaphoric gestures. Hum. Brain Mapp. 34 (7), 1696-1712.
Straube, B., Green, A., Weis, S., \& Kircher, T. (2012). A Supramodal Neural Network for Speech and Gesture Semantics: An fMRI Study. PLoS ONE, 7(11), e51207. doi:ARTN e51207 10.1371/journal.pone.0051207.

Straube, B., He, Y., Steines, M., Gebhardt, H., Kircher, T., Sammer, G., Nagels, A., 2013c. Supramodal neural processing of abstract information conveyed by speech and gesture. Front. Behav. Neurosci. 7, 120. https://doi.org/10.3389/fnbeh.2013.00120.

Straube, B., Wroblewski, A., Jansen, A., He, Y., 2018. The connectivity signature of cospeech gesture integration: The superior temporal sulcus modulates connectivity between areas related to visual gesture and auditory speech processing. Neuroimage 181, 539-549.

Thakkar, K.N., Peterman, J.S., Park, S., 2014. Altered Brain Activation During Action Imitation and Observation in Schizophrenia: A Translational Approach to Investigating Social Dysfunction in Schizophrenia. AJP 171 (5), 539-548.

Tzourio-Mazoyer, N., Landeau, B., Papathanassiou, D., Crivello, F., Etard, O., Delcroix, N., Joliot, M., 2002. Automated anatomical labeling of activations in SPM using a macroscopic anatomical parcellation of the MNI MRI single-subject brain. Neuroimage 15 (1), 273-289.

Van Overwalle, F., 2009. Social cognition and the brain: a meta-analysis. Hum. Brain Mapp. 30 (3), 829-858.

Vuilleumier, P., Armony, J.L., Driver, J., Dolan, R.J., 2001. Effects of attention and emotion on face processing in the human brain: an event-related fMRI study. Neuron 30 (3), 829-841.

Walther, S., Alexaki, D.D., Stegmayer, K.D.L., Vanbellingen, T., Bohlhalter, S., 2019. Conceptual disorganization impairs hand gesture performance in schizophrenia. Schizophr. Res.

Walther, S., Eisenhardt, S., Bohlhalter, S., Vanbellingen, T., Müri, R., Strik, W., Stegmayer, K., 2016. Gesture performance in schizophrenia predicts functional outcome after 6 months. Schizophr. Bull. 42 (6), 1326-1333.

Walther, S., Stegmayer, K., Sulzbacher, J., Vanbellingen, T., Müri, R., Strik, W., Bohlhalter, S., 2015. Nonverbal social communication and gesture control in schizophrenia. Schizophr. Bull. sbu222.

Walther, S., Vanbellingen, T., Müri, R., Strik, W., Bohlhalter, S., 2013. Impaired pantomime in schizophrenia: association with frontal lobe function. Cortex 49 (2), 520-527.

Wang, L., Chu, M., 2013. The role of beat gesture and pitch accent in semantic processing: An ERP study. Neuropsychologia 51 (13), 2847-2855.

Wensing, T., Cieslik, E.C., Müller, V.I., Hoffstaedter, F., Eickhoff, S.B., NicklJockschat, T., 2017. Neural correlates of formal thought disorder: An activation likelihood estimation meta-analysis. Hum. Brain Mapp. 38 (10), 4946-4965.

White, T.P., Joseph, V., Francis, S.T., Liddle, P.F., 2010. Aberrant salience network (bilateral insula and anterior cingulate cortex) connectivity during information processing in schizophrenia. Schizophr. Res. 123 (2-3), 105-115.

Witteman, J., Van Heuven, V.J.P., Schiller, N.O., 2012. Hearing feelings: A quantitative meta-analysis on the neuroimaging literature of emotional prosody perception. Neuropsychologia 50 (12), 2752-2763.

Wroblewski, A., He, Y., Straube, B., 2020. Dynamic Causal Modelling suggests impaired effective connectivity in patients with schizophrenia spectrum disorders during gesture-speech integration. Schizophr. Res. 216, 175-183.

Wurm, M.F., Caramazza, A., 2019. Distinct roles of temporal and frontoparietal cortex in representing actions across vision and language. Nat. Commun. 10 (1), 1-10. 